\title{
The complex structure of the disk around HD 100546 ^
}

\section{The inner few astronomical units}

\author{
M. Benisty ${ }^{1}$, E. Tatulli ${ }^{2}$, F. Ménard ${ }^{2}$, and M. R. Swain ${ }^{3}$ \\ 1 INAF-Osservatorio Astrofisico di Arcetri, Largo E. Fermi 5, 50125 Firenze, Italy \\ e-mail: benisty@arcetri.astro.it \\ 2 Laboratoire d'Astrophysique de Grenoble, CNRS-UJF UMR 5571, 414 rue de la Piscine, 38400 St Martin d'Hères, France \\ 3 Jet Propulsion Laboratory, California Institute of Technology, 4800 Oak Grove Drive, Pasadena, CA 91109, USA
}

Received 3 November 2009 / Accepted 7 January 2010

\section{ABSTRACT}

\begin{abstract}
Context. Disclosing the structure of disks surrounding Herbig AeBe stars is important to expand our understanding of the formation and early evolution of stars and planets. The first astronomical units of these disks in particular, because they are hot, dense, and subject to intense radiation field, hold critical clues to accretion and ejection processes, as well as planet formation in environment different than what prevailed around our own early Sun.

Aims. We aim at revealing the sub-AU disk structure around the $10 \mathrm{Myr}$ old Herbig Be star HD 100546 and at investigating the origin of its near and mid-infrared excess.

Methods. We used new AMBER/VLTI observations to resolve the $K$-band emission and to constrain the location and composition of the hot dust in the innermost circumstellar disk. Combining AMBER observations with photometric and MIDI/VLTI measurements from the litterature, we revisit the disk geometry using a passive disk model based on 3D Monte-Carlo radiative transfer (including full anisotropic scattering).

Results. We propose a model that includes a tenuous inner disk made of micron-sized dust grains, a gap, and a massive optically thick outer disk, that successfully reproduces the interferometric data and the SED. We locate the bulk of the $K$-band emission at $\sim 0.26 \mathrm{AU}$. Assuming that this emission originates from silicate dust grains at their sublimation temperature of $1500 \mathrm{~K}$, we show that micron-sized grains are required to enable the dust to survive at such a close distance from the star. As a consequence, in our best model, more than $40 \%$ of the $K$-band flux is related to scattering, showing that the direct thermal emission of hot dust is not always sufficient to explain the near-infrared excess. In the massive outer disk, large grains in the mid-plane are responsible for the mm emission while a surface layer of small grains allows the mid and far infrared excesses to be reproduced. Such vertical structure may be an evidence for sedimentation. The interferometric observations are consistent with a disk model that includes a gap until $\sim 13$ AU from the star and a total dust mass of $\sim 0.008$ lunar mass $\left(\sim 6.10^{23} \mathrm{~g}\right)$ inside it. These values together with the derived scale height $(\sim 2.5 \mathrm{AU})$ and temperature $(\sim 220 \mathrm{~K})$ at the inner edge of the outer disk $(r=13 \mathrm{AU})$, are consistent with recent CO observations.
\end{abstract}

Key words. accretion, accretion disks - radiative transfer - instrumentation: interferometers

\section{Introduction}

Extrasolar planets have been discovered at a regular pace since 1995. Late in 2008, five of them were imaged directly, two of them clearly being associated with the disk they formed in (Kalas et al. 2008; Lagrange et al. 2008). Yet, the exact process by which they form, and the timing of their formation within the disks remain unclear. There has been several interesting advances in the last years: direct imaging of debris disks, the discovery of fine structure in young disks likely due to gravitational interactions with bodies within or outside the disk, and finer sampling (spectroscopy) of the mid-infrared spectral energy distribution (SED). It is now clear that several processes are simultaneously acting on the disks at a given time. In the same time interval, it is expected that planets are actively forming.

In a series of multi-scale panchromatic studies (Doucet et al. 2007; Pinte et al. 2008b; Grady et al. 2009), we have initiated the study of several well documented disks surrounding

$\star$ Based on observations collected at the VLTI (ESO Paranal, Chile) with program 076.C-0851(B). pre-main-sequence stars. They will provide a testbed to study the conditions in which planets form. One of these stars is the late Herbig Be star, HD 100546 (B9.5Ve, 103pc; van den Ancker et al. 1997). Its circumstellar environment was first resolved in scattered light by Pantin et al. (2000), revealing the presence of a disk with an inclination of $\sim 50^{\circ}$. Coronagraphic imaging showed that the star is surrounded by a large scale envelope ( $1000 \mathrm{AU})$ and a disk extending up to $515 \mathrm{AU}$ whose brightness profile is asymmetric (Augereau et al. 2001; Grady et al. 2001). Its position in the HR diagram gives an estimated age larger than $10 \mathrm{Myr}$, supported by the presence of large fractions of crystalline silicates that suggest an evolved disk (Bouwman et al. 2003).

The SED of the system has been intensively studied. Compared to other HerbigAeBe stars, its specificity is to show a strong mid-infrared excess, peaking at $40 \mu \mathrm{m}$, compared to a rather weak 2-8 $\mu \mathrm{m}$ emission. To account for the deficit of NIR excess, Bouwman et al. (2003) suggested the presence of an inner cavity (empty region) within $10 \mathrm{AU}$, while fitting the SED with three optically thin concentric shells of different grain populations. Besides, support for a region of low density 
Table 1. Log of the observations, obtained on June 24, 2006, with the UT3-UT4 baseline.

\begin{tabular}{ccccc}
\hline \hline $\begin{array}{c}\text { Observation } \\
\text { number }\end{array}$ & $\begin{array}{c}\text { Projected } \\
\text { length }(\mathrm{m})\end{array}$ & $\begin{array}{c}\text { Position } \\
\text { angle }\left({ }^{\circ}\right)\end{array}$ & $\begin{array}{c}\text { Spectral } \\
\text { range }(\mu \mathrm{m})\end{array}$ & $\begin{array}{c}\text { Resolution } \\
(\text { mas })\end{array}$ \\
\hline$\# 1$ & 59.8 & 99.5 & $2.06-2.46$ & $7.1-8.5$ \\
$\# 2$ & 60.4 & 104.5 & " & $7.0-8.4$ \\
\hline
\end{tabular}

in dust grains is provided by the low circumstellar polarization in comparison with younger Herbig stars (Clarke et al. 1999). Spectroscopic observations also agree with this conclusion. STIS observations of $\mathrm{H}_{2}$ emission revealed the presence of a central cavity extending to 13 AU (Grady et al. 2005). Similarly, Acke \& van den Ancker (2006) concluded to the presence of a gap distant of $6.5 \mathrm{AU}$ from the star by studying the $6300 \AA[\mathrm{OI}]$ line. The last authors speculated that this gap (or secondary wall) is induced by a very low-mass stellar or planetary companion ( $20 M_{\text {Jup }}$ ). This could be the explanation for the pericenter asymmetry observed in the reflection nebulosity within the cavity by Grady et al. (2005) that could else not be due to chemistry effects (asumming that the density distribution is axisymmetric). The recent $\mathrm{CO}$ observations (Brittain et al. 2009; van der Plas et al. 2009) reported an extended emission with a large inner hole inside $\sim 11-13 \mathrm{AU}$, confirming the status of transitional disk given to HD 100546.

Nevertheless, the measured NIR excess as well as the presence of gas located from 0.5 to $60 \mathrm{AU}$ (Acke \& van den Ancker 2006; Martin-Zaïdi et al. 2008), imply that the inner AUs are not completely empty. In the mid-infrared wavelength range, the disk is resolved with mid-infrared spectro-interferometry (Leinert et al. 2004) and nulling interferometry showed that the radial temperature law in the few inner AUs (at $10 \mu \mathrm{m}$ and $20 \mu \mathrm{m}$ ) is not compatible with a continuously flared disk (Liu et al. 2003). Vinković et al. (2006) successfully reproduced the NIR SED using an optically thick disk with an inner radius at $0.45 \mathrm{AU}$, surrounded by an optically thin dusty enveloppe. All these results point toward structural differences between the inner and outer regions of the HD 100546 disk.

Constraining the spatial structure and physical conditions in the innermost disk is therefore a crucial issue. In particular, the exact position and geometry of the inner rim remain poorly constrained. NIR interferometric observations can achieve the spatial resolution needed to resolve this hot circumstellar material. The combination of angular and spectral resolution provided by AMBER at the ESO/VLTI allow us to directly resolve the circumstellar material located in the first AU of the disk and to address the question of its geometry. In this paper, our goal is to revisit the disk geometry with a special emphasis on its inner AU. The radiative transfer models we have calculated aim at reproducing both the interferometric and photometric observations.

The paper is organized as follow: in Sect. 2, we first describe the observations and the data reduction procedure; in Sects. 3 and 4, we present the interferometric results followed by the description of our disk models. Finally, we discuss our results in Sect. 5 .

\section{Observations and data processing}

HD 100546 was observed at the Very Large Telescope Interferometer (VLTI; Haguenauer et al. 2008) with the AMBER instrument on 24 April 2006. AMBER simultaneously combines three beams in the near infrared $H$ and $K$ bands using spatial
Table 2. Stellar and calibrator properties.

\begin{tabular}{cccccc}
\hline \hline Star & $V$ & $K$ & $H$ & Sp. Type & $\begin{array}{c}\text { Diameter } \\
{[\mathrm{mas}]}\end{array}$ \\
\hline HD 100546 & 6.7 & 5.4 & 5.9 & B9Vne & - \\
HD 101531 & 7.3 & 4.2 & 4.3 & K1III & $0.6 \pm 0.1$ \\
\hline
\end{tabular}

filtering and spectral dispersion (Petrov et al. 2007). It therefore provides spectrally dispersed visibilities and closure phases. In the following, we present observations that were obtained with three 8-m unit telescopes (UT1, UT3, UT4) and AMBER in its low spectral resolution mode $(R \sim 30)$. In addition to HD 100546, a calibrator target of known diameter (HD 101531) was observed. Its properties can be found in Table 2. The observations were achieved under good weather conditions (seeing of $\sim 0.8^{\prime \prime}$ and coherence time of $\sim 5 \mathrm{~ms}$ ) and without fringe tracker. The data consist of respectively, 8 and 5 exposures of 1000 frames each, for HD100546 and its calibrator.

The data reduction was performed following standard procedures described in Tatulli et al. (2007), using the amdlib package, release 2.99 , and the yorick interface, provided by the Jean-Marie Mariotti Center (JMMC). Raw spectrally dispersed visibilities and closure phases are extracted for all the frames of each observing file. A selection process among them is necessary to avoid the effects of instrumental jitter and unsatisfactory light injection. Various selection procedures were applied, including selection based on the fringe signal-to-noise ratio (SNR) and on the fringe optical path difference (OPD; i.e. piston). Although no difference in absolute values was found between them, the best accuracy was obtained with a selection of $20 \%$ of the best exposures based on the fringe SNR. This criteria takes into account both the flux level and the fringe contrast (or visibility). Among all the measurements obtained on the three baselines, only the ones corresponding to the shortest baseline (UT3-UT4) in $K$-band, led to SNR above 1.5. Consequentely, all H-band measurements, $K$-band data corresponding to UT1-UT3 and UT1-UT4 baselines, and all the closure phases, were rejected. The remaining files have SNR ranging from 2.3 to 5.8. The same selection was applied to both the scientific star and calibrator measurements in order to guarantee an uniform data reduction. The attenuation in fringe contrast due to additional piston with respect to the zero OPD was corrected following Tatulli et al. (2007). Finally, calibration of the AMBER+VLTI instrumental transfer function was done using measurements of HD 101531, after correcting for its diameter. The errors on the calibrated spectral visibilities include the statistical error obtained when averaging individual frames, the error on the diameter of the calibration star, and the dispersion of the transfer function along the calibrator measurements. The last element is the one that dominates the error budget. After data processing, the final data set consists in 2 measurements on UT3-UT4 baseline, that are spectrally dispersed in $13 \mathrm{~K}$-band spectral channels. Therefore, in total, we obtained 26 visibilities in the [2.06-2.46] $\mu \mathrm{m}$ wavelength range. A summary can be found in Table 1 .

\section{Results}

\subsection{Visibilities}

Figure 1, left, shows the measured visibilities after data processing. They are plotted against the spatial frequency, $B / \lambda$, with $B$ the projected baseline length and $\lambda$, the wavelength of the observations. The spatial frequency is the inverse of the resolution 
M. Benisty et al.: The complex structure of the disk around HD 100546
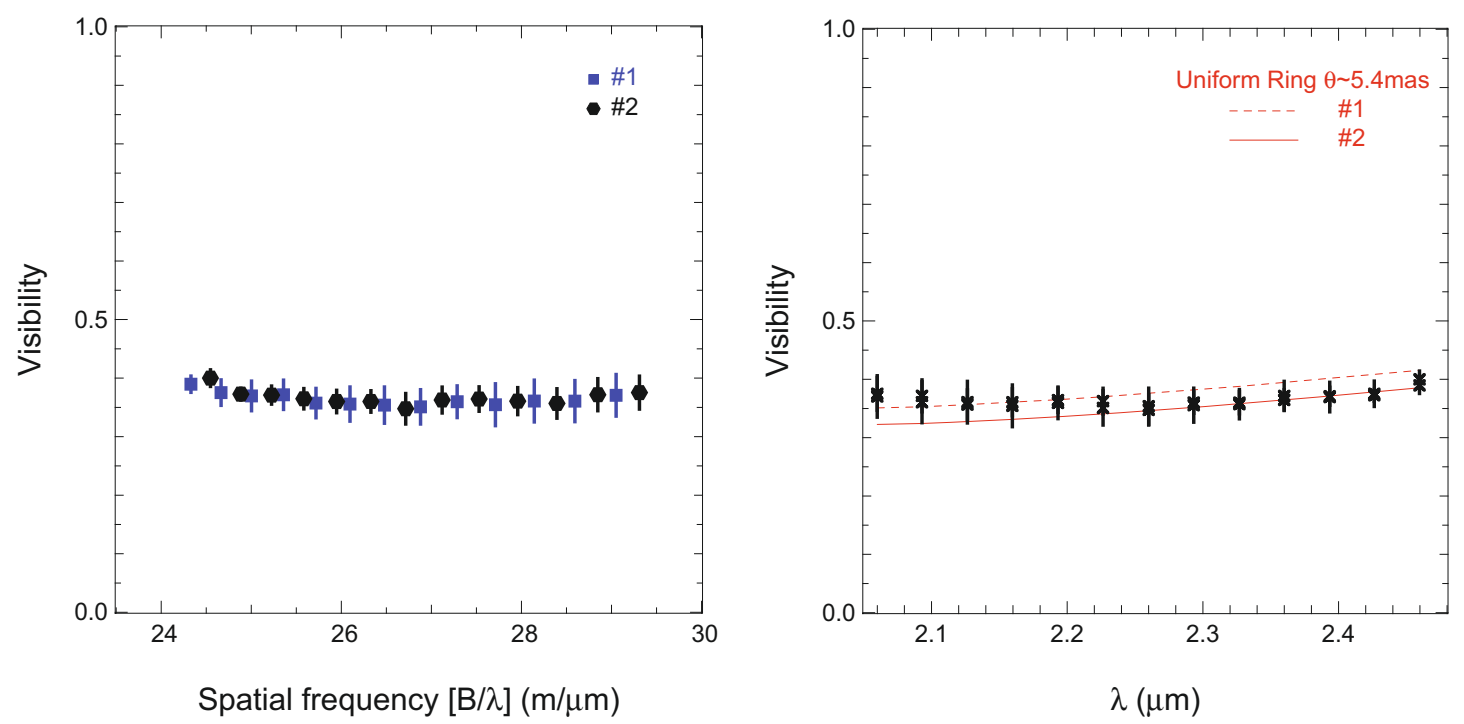

Fig. 1. Left: measured visibilities plotted against spatial frequency. The two different measurements (\#1, \#2) are shown with distinct symbols. Right: the visibilities (black crosses) are shown against wavelength and are compared to the predictions of a ring of uniform brightness. The red full and dashed lines give these predictions for the two measurements (i.e. at two distinct projected baselines).

achieved by the interferometer and takes into account the wavelength, the projected baseline, and consequentely, the direction on the sky. It can be expressed in unity $[\mathrm{m} / \mu \mathrm{m}]$ or in cycles per milli-arcsecond. It can be immediately seen that the circumstellar matter around HD 100546 is resolved at the level of a few milli-arcseconds, because the visibility is well below 1 .

To derive basic characteristics of the NIR emission, one can consider a simple geometrical model of a ring of uniform brightness that roughly accounts for hot dust located in the inner rim of a dusty disk. The model visibilities $V_{\text {mod }}$ can be written :

$V_{\mathrm{mod}}=\frac{V_{*}+V_{\mathrm{ex}} * F_{\mathrm{ex}} / F_{*}}{1+F_{\mathrm{ex}} / F_{*}}$

with $V_{*}=1$ (unresolved central star) and $F_{\text {ex }} / F_{*}$, the ratios of the NIR excess to the stellar flux. This ratio, $\sim 2.2$, is estimated at each AMBER wavelength, using a Kurucz model for the central star emission at $T=10500 \mathrm{~K}$. With this model, the extension of the $K$-band emission is found to be $\sim 5.4$ mas (i.e. a radius of $\sim 0.28 \mathrm{AU}$ ) at $d=103 \mathrm{pc}$. The observed chromaticity of the visibilities can be compared to the predictions of this model. Figure 1, right, shows the spectral visibilities against wavelength. The visibility curve is almost flat with wavelength, while the uniform brightness ring predicts a slightly steeper slope.

HD 100456 was also observed by Leinert et al. (2004) in the mid-infrared with the MIDI instrument at VLTI. These observations led to a single-dish image at $8.7 \mu \mathrm{m}$ and to visibility measurements (at $74 \mathrm{~m}$ baseline length). In Sect. 4, we use this image and the corresponding $8.7 \mu \mathrm{m}$ visibility.

\subsection{Photometry}

We retrieve from the litterature photometric measurements from the UV to the millimetric wavelengths to produce a complete SED (2MASS, IRAS, ISO; Ardila et al. 2007; Henning et al. 1994; Grady et al. 2001). Moreover, because young stars are highly variable in the NIR wavelengths (Sitko et al. 2008), and since modelling of the observations relies on these photometric values, it is useful to provide an estimate of the NIR emission directly from the interferometric measurements. We were able to extract the $K$ band photometry from the three AMBER photometric channels. Following Acke et al. (2008), for each file, we summed the flux in each channel over all frame and calibrated this value with the total flux in the corresponding channel for the calibrator. We applied a photospheric model for the calibrator star in order to convert it in flux units and obtain $5.33 \pm 0.08$ as the $K$ band magnitude, consistent with the 2MASS values.

\section{Modelling}

Since it has been long established that HD 100546 is surrounded by a disk (Augereau et al. 2001), in this section, we use disk models to describe its circumstellar environment. It is not possible to unambiguously estimate the disk inclination and position angle from a single-baseline interferometric measurement, therefore, we adopt the previously published values of $42^{\circ}$ and $145^{\circ}$, respectively (Ardila et al. 2007). Besides, we consider a total disk mass of $5 \times 10^{-4} M_{\odot}$, inferred by $1.3 \mathrm{~mm}$ imaging (Henning et al. 1994).

\subsection{The radiative transfer code MCFOST}

We used the Monte Carlo-based 3-D radiative transfer code, MCFOST, to produce disk images and SED (Pinte et al. 2006). The temperature distribution is calculated using the immediate re-emission concept of Bjorkman \& Wood (2001) but with a continuous deposition of energy to estimate the mean intensity (Lucy 1999). In this paper, we used a cylindrical grid, with an adaptive mesh at the inner edge (based on the opacity gradient) so as to properly sample the inner radius of the disk. The radiation field and temperature structure estimated by the Monte Carlo runs are used to produce SEDs by calculating the formal solution of the radiative transfer equation along rays (ray-tracing method), as well as images. Visibilities are computed by taking the Fourier transform of the latter, at the specific spatial frequencies sampled by the observations. The code has been extensively tested and its performances compared to other radiative transfer codes (Pinte et al. 2009).

We assume that the disk is constituted of silicate dust grains only (Pollack et al. 1994), and is in hydrostatic equilibrium. Its 

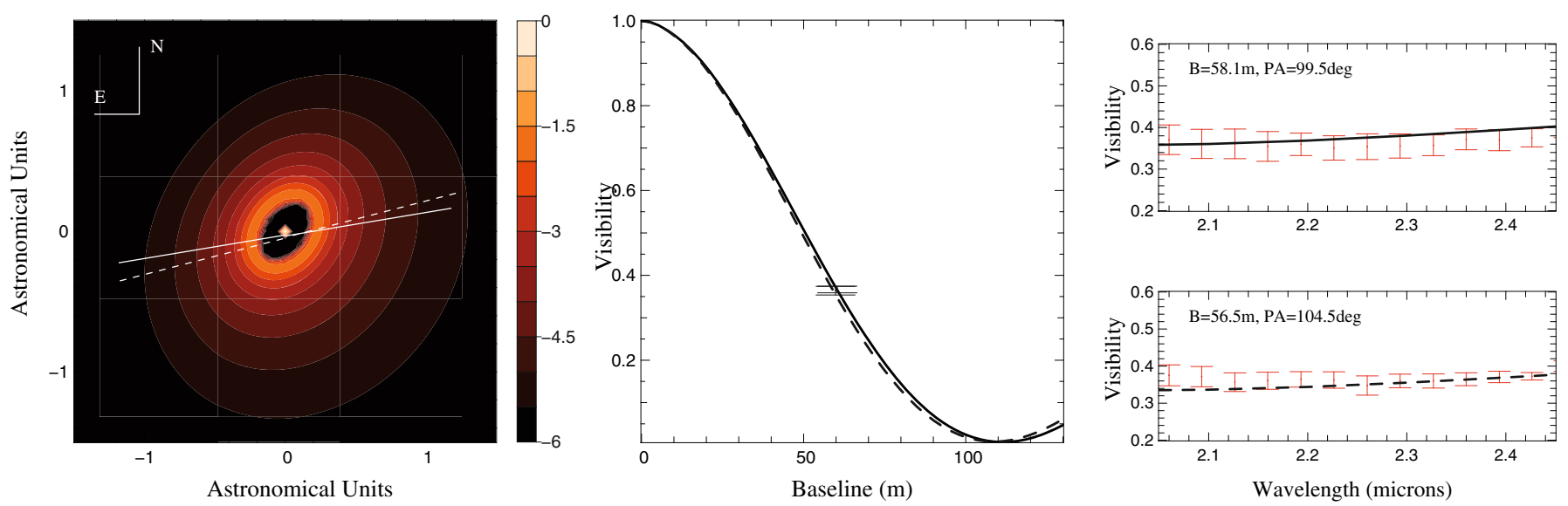

Fig. 2. Left: $K$-band model image of HD 100546. The full and dashed lines indicate the position angles of the observations $\left(\mathrm{PA}=99.5^{\circ}\right.$ and $\mathrm{PA}=104.5^{\circ}$, respectively). Middle: model visibilities, as a function of baseline length. The broad band $K$-band AMBER visibilities are overplotted. Right: spectrally dispersed AMBER visibilities versus wavelength, for both measurements (upper and lower pannels respectively). The spectral profiles of the model visibilities, as derived from our best model, are added.

structure is parametrized by power laws, so that the surface density $\Sigma$, and the scale height $H$ can be expressed as varying with radius, $r$, following :

$\Sigma(r)=\Sigma_{100 \mathrm{AU}}\left(\frac{r}{100 \mathrm{AU}}\right)^{q}$

$H(r)=\mathrm{H}_{100 \mathrm{AU}}\left(\frac{r}{100 \mathrm{AU}}\right)^{\beta}$

$\beta$ is the flaring exponent. $\Sigma_{100 \mathrm{AU}}$ and $H_{100 \mathrm{AU}}$ are the values of the surface density and the height scale at a radius of $100 \mathrm{AU}$. The dust grain sizes, $a$, are distributed following a power law $a^{-3.5}$ (ISM), with a varying between the minimum and maximum sizes, $a_{\min }$ and $a_{\max }$ respectively. In addition, we fix the total dust mass $\left(M_{\text {dust }}\right)$ of the disk, that is delimited by its inner and outer radii $\left(R_{\text {in }}\right.$ and $\left.R_{\text {out }}\right)$.

The disk of HD 100546 is well known and its geometry and content have been estimated by several authors (Grady et al. 2001; Bouwman et al. 2003; Brittain et al. 2009). We will therefore not provide a detailed re-analysis of all the disk structural parameters. To the contrary, using in spirit the results of previous studies, we will construct a disk model that also includes a large gap. However, with the help of interferometric measurements, and complete radiative transfer including scattering, we will revisit the morphology and mass content of the inner disk, as well as the position of the gap, in a global picture.

\subsection{The disk model}

The SED constrains the mass-temperature law for the dust. The near-infrared emission in excess of the photosphere is due to hot material at $\sim 1500 \mathrm{~K}$, and must result from a small amount of dust in an optically thin inner disk. The large mid and far-infrared excesses are due to dust at a temperature of $\sim 200 \mathrm{~K}$, while large and cold grains in the mid-plane of the disk must be responsible for the emission at longer wavelengths. The SED gives integrated flux informations but provides little morphological informations on the dust spatial distribution.

In this section, we propose a disk model that successfully accounts for the emission at all wavelengths, as well as for the AMBER and MIDI visibilities (see Figs. 2, 4 and 5). However, our model does not attempt to match mineralogical features. As pointed out by Bouwman et al. (2003), continuous flared disk models can not reproduced the SED as they produce either too much or too little flux in the near and mid-infrared wavelengths, respectively. The model includes an optically thin inner disk, a gap and an optically thick outer disk. The model parameters can be found in Table 3, including for each zone, the mass $\left(M_{\text {dust }}\right)$, the inner and outer radii ( $R_{\text {in }}, R_{\text {out }}$ respectively), the scale height at $100 \mathrm{AU}\left(H_{100 \mathrm{AU}}\right)$, the flaring and surface density exponents $(\beta, q$, respectively) as well as the minimum and maximum grain sizes ( $a_{\min }, a_{\max }$, respectively).

The inner disk: the AMBER/VLTI measurements can locate the bulk of the emission in the $K$-band at $\sim 0.25-0.30$ AU. This puts a strong constraint on the location of the disk inner edge and consequentely on the silicate dust grains sizes. The tenuous inner disk has a total dust mass of $3 \times 10^{-10} M_{\odot}$ and starts at a radius of $\sim 0.26 \mathrm{AU}$, a distance inferred from sublimation of micron-sized silicate grains (specifically, with sizes ranging from 0.1 to $5 \mu \mathrm{m}$ ) at $\sim 1500 \mathrm{~K}$. Its outer radius is not well constrained by the AMBER observations because it is fully resolved, and we fix it at $\sim 4 \mathrm{AU}$. Here, all the model calculations are done assuming that the dust grains are at the same equilibrium temperature with the stellar radiation field which may not be exactly the case in the tenuous optically thin inner regions. Although a more detailed calculation, that requires determination of the dust-to-gas ratio is beyond the goal of this paper, we stress that there may not be a specific inner radius but a range of inner radii, depending on the grain sizes.

The gap: after computing numbers of models, we confirm the need for a very low density region (e.g. a gap) to reproduce the lack of emission in the $3-10 \mu \mathrm{m}$ wavelength range. The MIDI/VLTI measurements provide a complementary information on the spatial distribution of the dust in the disk. At the achieved angular resolution at $\sim 8.7 \mu \mathrm{m}$ (24 mas i.e. $2.4 \mathrm{AU})$, the innermost disk is not resolved but the outer disk is fully resolved. The $8.7 \mu \mathrm{m}$ observations therefore bring a strong constraint on the flux ratio, between these two elements of the disk. In the model, the gap starts at $4 \mathrm{AU}$ until $13 \mathrm{AU}$, a radius consistent with spectroscopic results (Grady et al. 2005; Brittain et al. 2009).

The outer disk and its surface layers: the model massive outer disk forms at $13 \mathrm{AU}$ and extends up to $350 \mathrm{AU}$. It contains most of the disk mass and holds large dust grains (from $1 \mu \mathrm{m}$ 

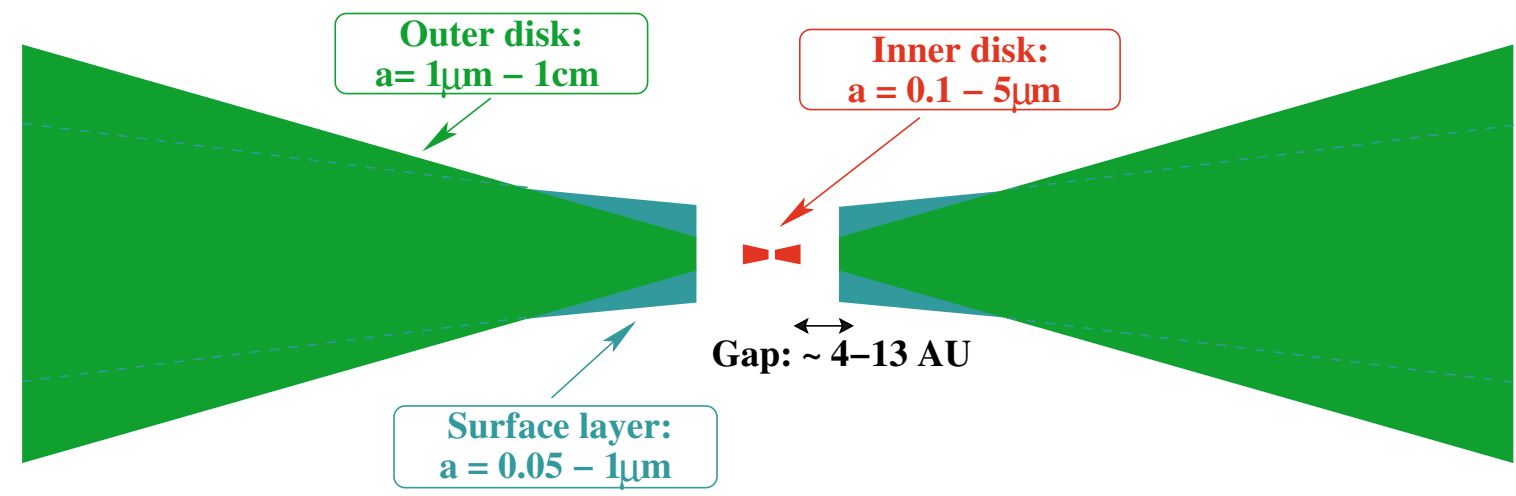

$\sim 350 \mathrm{AU}$

Fig. 3. Schematic view of our best disk model, that includes different zones: an inner disk with micron-sized silicate grains, a gap, a massive outer disk with small grains in an upper layer.
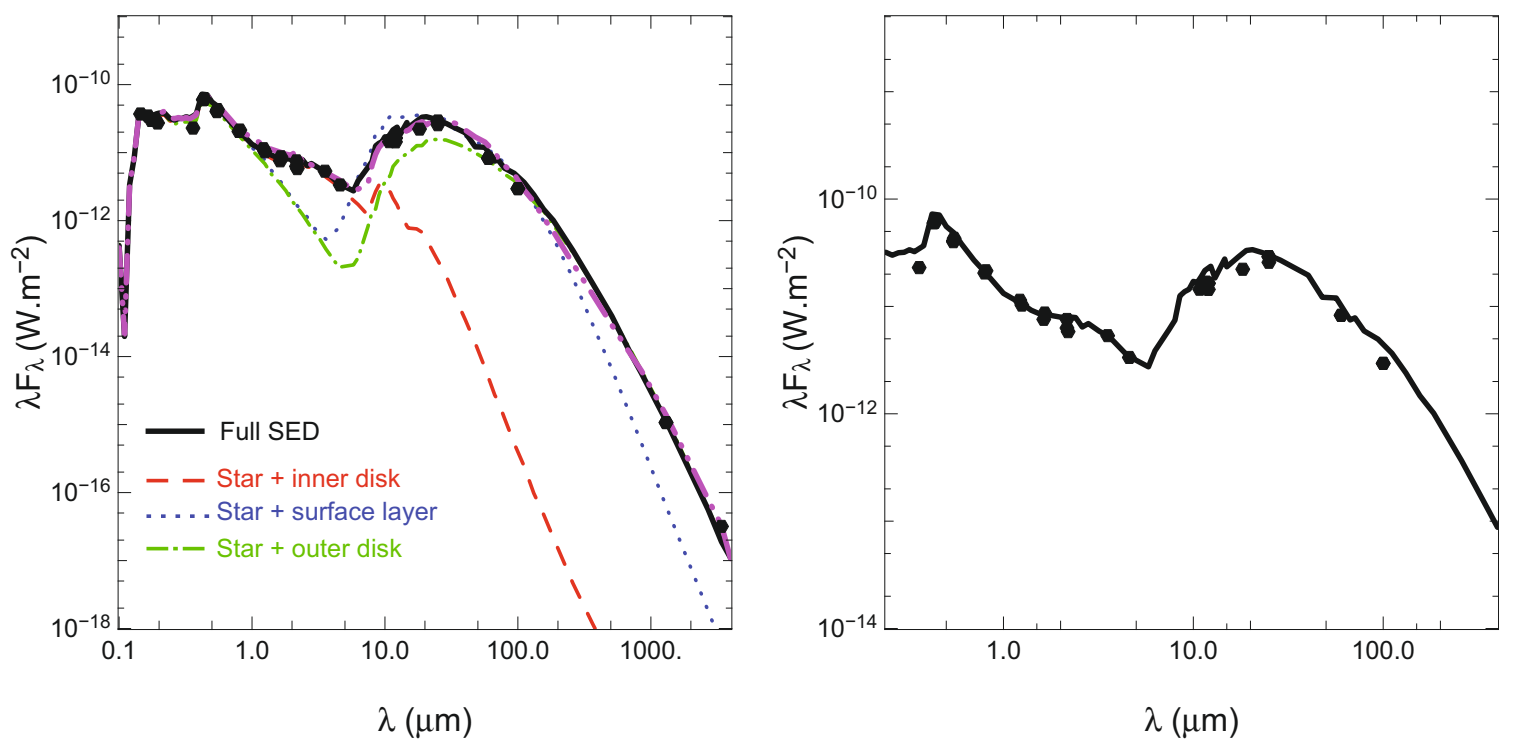

Fig. 4. Left: measured SED (circles) for HD 100546, compared to the predictions of our best model (black full line). The individual contributions of each disk zone to the SED are shown (inner disk, red dashed curve; surface layer, blue dotted curve; outer disk, green dash-dotted line). A similar fit to the SED can be obtained with a different disk model (magenta line). Right: zoom in the near and mid-infrared wavelengths.

to $10 \mathrm{~mm})$. To account for the mid and far-infrared excesses, we include a population of small grains (i.e. 0.05 to $1 \mu \mathrm{m}$ ) at the surface of the outer disk, at distances of a few tens of AUs (here, from 13 to $50 \mathrm{AU}$; see Table 3). The latter are not precisely constrained by the observations, as the only requirement is to have a specific amount of small grains emitting at a few tens of AUs.

The entire set of parameters used in this model is given in Table 3, and a schematic view of the disk model is provided in Fig. 3. In Fig. 4, we report the contributions from each part of the disk to the total flux. The inner disk (red dashed line) produces enough emission to fit the NIR excess while the small grains in the surface layers (blue dotted line) produce the major contribution to the large mid and far infrared excess. Finally, the large grains in the outer disk are responsible for the flux at long wavelength (green dash-dotted line). The predictions of the entire model are in good agreement with the observations (full black line).

For this model, images and visibilities were computed at the AMBER wavelengths. Figure 2 (middle) shows the visibility profile as function of the projected baseline length. This model succesfully reproduces the interferometric observations in addition to the SED: the spatial extent of its $K$-band emission and its inner structure are in good agreement with the data. The chromaticity in the $K$-band is also well reproduced with the radial temperature profile of the disk model (Fig. 2, right). In the midinfrared, we focus our modelling on the continuum emission at $8.7 \mu \mathrm{m}$ and do not attempt to reproduce any spectral feature. Figure 5 (left) gives the model image at $8.7 \mu \mathrm{m}$ that clearly shows an elongated disk structure, in agreement with the one obtained by Leinert et al. (2004) (Fig. 5, middle). From a simple $2 \mathrm{D}$ fit, we obtain $\sim 21 \mathrm{AU}$ and $\sim 14 \mathrm{AU}$ as the disk major and minor axis, respectively, in good agreement with the values derived by Leinert et al. (2004) $(29 \pm 4$ AU and $19 \pm 9$ AU respectively) and compatible with the results from nulling interferometry (24 AU; Liu et al. 2003). Figure 5, right, shows the model visibilities at $8.7 \mu \mathrm{m}$, compared to the MIDI measurement. At long baselines, the outer disk is entirely resolved and one has access to the unresolved flux contribution of the inner disk to the total $8.7 \mu \mathrm{m}$-flux. At a $74 \mathrm{~m}$-baseline, our model can well 

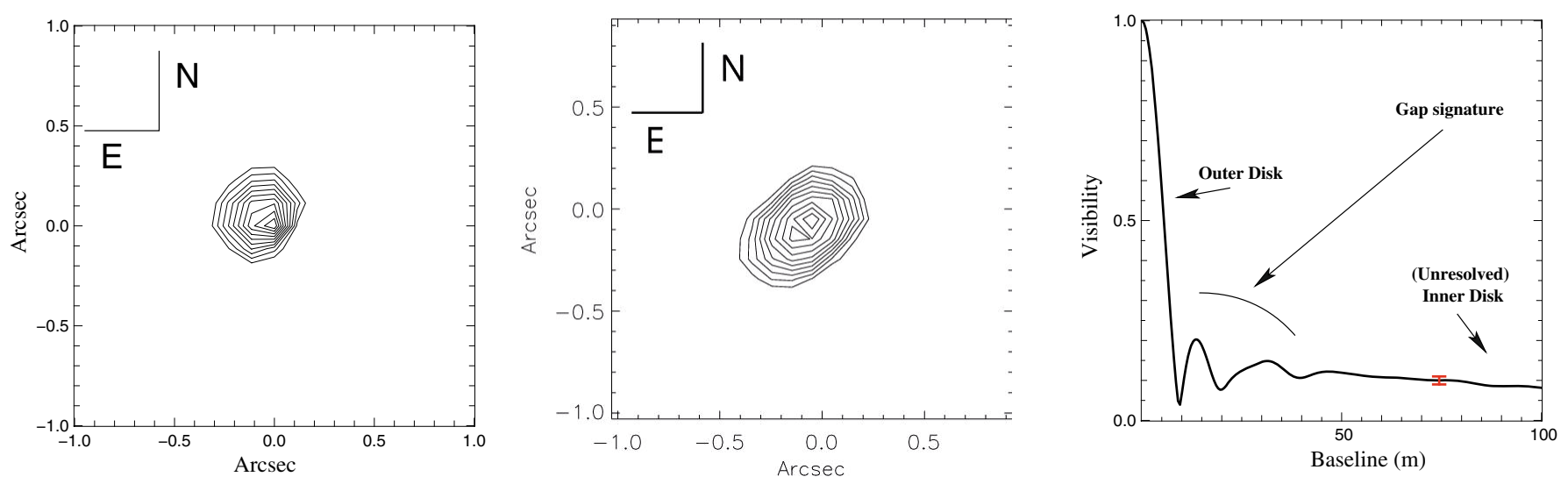

Fig. 5. Left: $8.7 \mu \mathrm{m}$ continuum image of HD 100546 predicted by our best model and oriented along a $145^{\circ}$ position angle. Middle: $8.7 \mu \mathrm{m}$ continuum image obtained with a single UT at VLT (Leinert et al. 2004). For both images, the contour levels are linearly spaced from $100 \%$ to $25 \%$ as the fourth root of the image brightness. Right: visibility curve calculated for our best model (solid line) at $8.7 \mu \mathrm{m}$. The MIDI measurement obtained by Leinert et al. (2004) is shown with the error bar.

Table 3. Parameters of our best model.

\begin{tabular}{lcccc}
\hline \hline & & Inner disk & Surface layer & Outer disk \\
\hline$M_{\text {dust }}$ & {$\left[M_{\odot}\right]$} & $3 \mathrm{e}-10$ & $6 \mathrm{e}-5$ & $5 \mathrm{e}-4$ \\
$R_{\text {in }}$ & {$[\mathrm{AU}]$} & 0.26 & 13 & 13 \\
$R_{\text {out }}$ & {$[\mathrm{AU}]$} & 4 & 50 & 350 \\
$H_{100 \mathrm{AU}}$ & {$[\mathrm{AU}]$} & 6 & 12 & 12 \\
$\beta$ & & 1 & 0.5 & 1.125 \\
$q$ & & -1 & -1 & -1 \\
$a_{\min }$ & {$[\mu \mathrm{m}]$} & 0.1 & 0.05 & 1 \\
$a_{\max }$ & {$[\mu \mathrm{m}]$} & 5 & 1 & 10000 \\
\hline
\end{tabular}

Table 4. Contributions from the different types of emission to the total flux.

\begin{tabular}{ccccc}
\hline \hline Model with & $F_{\text {dir,st }} / F_{\text {tot }}$ & $F_{\text {sc,st }} / F_{\text {tot }}$ & $F_{\text {dir,th }} / F_{\text {tot }}$ & $F_{\text {sc,th }} / F_{\text {tot }}$ \\
\hline$R_{\text {in }}=0.26 \mathrm{AU}$ & 19.6 & 4.1 & 38.0 & 38.3 \\
$R_{\text {in }}=0.40 \mathrm{AU}$ & 16.7 & 1.8 & 64.4 & 17.1 \\
\hline
\end{tabular}

account for this flux ratio. Our model predicts that, at short baselines $(\leq 10 \mathrm{~m})$, the outer disk is resolved as the steep decrease of visibility attests, while for intermediate baselines, the sharp edge of the gap produces a clear signature. Mid-infrared interferometry therefore appears well suited to probe the presence of a gap, as the strong gap signature can easily be detected using moderate VLTI baselines.

\section{Discussion}

In this paper, we propose a model of the disk surrounding HD 100546 that successfully reproduces the SED, AMBER and MIDI interferometric observations. We note that equally good fits to the SED can be obtained with other models, similar in spirit to the one we propose (i.e. with three distinct disk regions) but with different spatial distribution. In particular, Bouwman et al. (2003) suggested a solution to reproduce the SED, that includes a population of small grains distributed from 0.3 to 9.8 AU, a more massive part with small grains from 9.8 to $43 \mathrm{AU}$, and a population of large dust grains from 28 to $380 \mathrm{AU}$, with a grain size distribution $n(a) \propto a^{-2}$. Different species are used to study the mineralogy of the dust, including refractory grains, such as iron. Using the same grain sizes distribution, but for silicate only, we could produce a good fit to the SED (see Fig. 4, left, magenta curve). To account for the NIR emission, the small grains are nevertheless required to be hotter than the tabulated evaporation temperature for silicate (i.e. $2100 \mathrm{~K}$ instead of $1500 \mathrm{~K}$ ). Besides, they induce a slightly different location for the bulk of the $K$-band emission (i.e. $0.4 \mathrm{AU}$ ) which does not match the AMBER visibilities. We therefore confirm here that spatially resolved informations are needed to speculate on the disk structure and properties, as fitting the SED alone is highly ambiguous.

The importance of scattering: an interesting side effect is the amount of scattered light induced in different models. At each wavelength, the total emission $\left(F_{\text {tot }}\right)$ is constituted of 4 distinct contributions: the stellar light that directly reaches the observer $\left(F_{\text {dir,st }}\right)$; the stellar light that is scattered by the disk $\left(F_{\mathrm{sc}, \mathrm{st}}\right)$; the direct thermal emission from dust grains $\left(F_{\mathrm{dir}, \mathrm{th}}\right.$; i.e. absorbed and then re-emitted photons) and finally, the scattered thermal light $\left(F_{\text {sc,th }}\right.$; i.e. absorbed, re-emitted and then scattered photons). Micron-sized silicate grains located at $0.26 \mathrm{AU}$ are good scatterers, since their sizes are of the order of the AMBER wavelengths. In our model, the scattered thermal light contributes to $\sim 38 \%$ of the total $K$-band emission, about as much as the direct thermal flux. On the contrary, the model with small grains in the inner disk and an inner radius at $0.4 \mathrm{AU}$, predicts a dominant contribution from direct thermal light $(\sim 64 \%)$. Table 4 reports the individual flux contributions to the total $K$ band emission $\left(F_{\text {tot }}\right)$. The index "dir" or "sc" indicates whether it is direct or scattered light, while the index "st" or "th" designate stellar and thermal light, respectively. Assuming that the disk is made of silicate grains only, the fit to the AMBER data constrains the grain size and consequently the amount of scattered light. This rules out solutions where only direct thermal emission from the dust is assumed. This means that scattered light can have a major effect in the overall energy budget in the NIR, and a strong impact on the inner disk parameters inferred from modelling. This was already showed and taken into account in modelling observations of solar-mass young stars, as previously shown by Pinte et al. (2008a), but this is the first time that such case is made for a Herbig Be star. This conclusion in fact contrasts with the usual assumption made to interpret NIR interferometric results in Herbig AeBe stars, i.e. that the NIR excess emission is due only to direct thermal emission from hot dust located at the inner edge of a disk (Dullemond et al. 2001). In fact, scattering or additional emitting components (such as gas Isella et al. 2008; or refractory dust grains; Benisty et al. 2009) seem to be needed to reproduce recent spectro-interferometric 
observations of Herbig AeBe stars. Whether this is relevant for all the objects of this class should be validated on simpler cases.

Evidence for sedimentation? The need for small grains at the surface layers and for large grains in the bulk of the disk can be interpreted as resulting from grain growth and dust settling. A toy model was computed with dust grain sizes ranging from 0.05 to $3000 \mu \mathrm{m}$, a standard flaring $(\beta=1.125)$, but considering a larger scale height (36 AU at $100 \mathrm{AU}$ ) and a strong dust settling. The predicted emission similarly fits the SED. Considering the age of the system, the disk is very likely evolved and vertical stratification could well be happening.

A gap and a tenuous inner disk: modelling of the SED requires the presence of a gap. In fact, a continuous disk model can fit the AMBER data and the flux at $2.2 \mu \mathrm{m}$, but it produces too much flux at $8.7 \mu \mathrm{m}$. Similarly, a continuous disk model that would fit the MIDI data and the $8.7 \mu \mathrm{m}$ flux, would in turn predict too little flux in the NIR. This confirms the need for a gap, or at least, for a strong discontinuity in the radial density profile that would produce the needed amounts of flux at specific locations.

If the need for this density discontinuity is clear, its size is nevertheless very model-dependent. In fact, we can not determine the position of the outer radius of the inner disk with precision, because it is fully resolved by AMBER. The MIDI observations constrain the flux ratio between the inner and outer disks: when our inner disk extends up to $\sim 4 \mathrm{AU}$, the outer disk starts at $\sim 13$ AU. A change in the inner disk geometry would affect this estimate. In our model, it is constrained by the temperature, hence position, of the emitting particles. Considering the typical grain size distributions given in Table 3, our estimate is in good agreement with the values inferred from previous studies (Brittain et al. 2009; Grady et al. 2005).

Bouwman et al. (2003) required the disk to be more puffed up at $10 \mathrm{AU}$ than prediced by a standard flared disk at the same radius to capture and re-emit enough light. They use $3.5 \mathrm{AU}$ as scale height for dust at $\sim 200 \mathrm{~K}$ which is much more than expected for gas at the same temperature (i.e. $1 \mathrm{AU}$ ). Brittain et al. (2009) found that the $\mathrm{CO}$ disk extends from $13 \pm 6 \mathrm{AU}$ and that at the inner edge of the disk, the gas is much hotter $(\sim 1400 \mathrm{~K})$ than the dust at the same radius. As the authors suggest, this can be explained if there is a low density region in the upper disk atmosphere, that results in a scale height of $\sim 3 \mathrm{AU}$. In our model, the scale height of the outer disk at its inner edge is $\sim 2.5 \mathrm{AU}$, due to the small grains in the surface layers, a value in agreement with the predictions of Brittain et al. (2009).

Moreover, Bouwman et al. (2003) predicted a total dust mass of $10^{25} \mathrm{~g}$ inside the gap. This led Brittain et al. (2009) to deduce from their estimate of the mass of gas in the inner disk $\left(\leq 3 \times 10^{25} \mathrm{~g}\right)$ that the gas-to-dust ratio is $\sim 3$. Our model requires a total dust mass of $3 \times 10^{-10} M_{\odot}\left(6 \times 10^{23} \mathrm{~g}\right)$ i.e. a gas-to-dust ratio of $\sim 50$. We therefore conclude that these estimates are highly model-dependent and should be considered with care, pending a direct measurement of the dust content of the inner disk. The small accretion rate, inferred from UV spectroscopy (Deleuil et al. 2004; Grady et al. 2005) - a few times $10^{-9} M_{\odot} \cdot \mathrm{yr}^{-1}-$ is enough to empty the cavity in a few decades, yet we are observing dust in the inner disk at an age $\geq 10$ Myr. Either we are observing HD 100546 at a very special moment in its evolution or the inner disk is being replenished. We note that a putative large orbiting body, a Jupiter-like planet located at about $10 \mathrm{AU}$, has the capacity to carve the disk and lead to such a surface density discontinuity at $\sim 13$ AU, while allowing some material to flow in continuously across the gap all the way to the star (Varnière et al. 2006).

This transitional disk will be a primary target for ALMA that will enable direct determination of the gap extent, by accurately probing the gas surface density profile. Before then, additional VLTI data with three telescopes, allowing phase closure detections, will add new constraints such as the asymmetry of the inner disk rim, the co-planarity of the inner and outer disks (Grady et al. 2009), and whether the star is off-center with respect to the gap (Grady et al. 2005).

Acknowledgements. We wish to thank ANR through contract ANR-07-BLAN0221, the PNPS program of CNRS/INSU (France), and INAF (grant ASI-INAF $\mathrm{I} / 016 / 07 / 0)$. We acknowledge the anonymous referee for his suggestions that improved the quality of the manuscript.

\section{References}

Acke, B., \& van den Ancker, M. E. 2006, A\&A, 449, 267

Acke, B., Verhoelst, T., van den Ancker, M. E., et al. 2008, A\&A, 485, 209 Ardila, D. R., Golimowski, D. A., Krist, J. E., et al. 2007, ApJ, 665, 512 Augereau, J. C., Lagrange, A., Mouillet, D., \& Ménard, F. 2001, A\&A, 365, 78 Benisty, M., Natta, A., Isella, A., et al. 2010, A\&A, 511, A74 Bjorkman, J. E., \& Wood, K. 2001, ApJ, 554, 615

Bouwman, J., de Koter, A., Dominik, C., \& Waters, L. 2003, A\&A, 401, 577 Brittain, S. D., Najita, J. R., \& Carr, J. S. 2009, ApJ, 702, 85

Clarke, D., Smith, R. A., \& Yudin, R. V. 1999, A\&A, 347, 590

Deleuil, M., Lecavelier des Etangs, A., Bouret, J., et al. 2004, A\&A, 418, 577

Doucet, C., Habart, E., Pantin, E., et al. 2007, A\&A, 470, 625 Dullemond, C. P., Dominik, C., \& Natta, A. 2001, ApJ, 560, 957 Grady, C. A., Polomski, E. F., Henning, T., et al. 2001, AJ, 122, 3396 Grady, C. A., Woodgate, B., Heap, S. R., et al. 2005, ApJ, 620, 470 Grady, C. A., Schneider, G., Sitko, M. L., et al. 2009, ApJ, 699, 1822 Haguenauer, P., Abuter, R., Alonso, J., et al. 2008, in SPIE Conf. Ser., 7013 Henning, T., Launhardt, R., Steinacker, J., \& Thamm, E. 1994, A\&A, 291, 546 Isella, A., Tatulli, E., Natta, A., \& Testi, L. 2008, A\&A, 483, L13

Kalas, P., Graham, J. R., Chiang, E., et al. 2008, Science, 322, 1345 Lagrange, A., Gratadour, D., Chauvin, G., et al. 2009, A\&A, 493, 121 Leinert, C., van Boekel, R., Waters, L. B. F. M., et al. 2004, A\&A, 423, 537 Liu, W. M., Hinz, P. M., Meyer, M. R., et al. 2003, ApJ, 598, L111 Lucy, L. B. 1999, A\&A, 345, 211

Martin-Zaïdi, C., Deleuil, M., Le Bourlot, J., et al. 2008, A\&A, 484, 225

Pantin, E., Waelkens, C., \& Lagage, P. O. 2000, A\&A, 361, L9

Petrov, R. G., Malbet, F., Weigelt, G., et al. 2007, A\&A, 464, 1

Pinte, C., Ménard, F., Duchêne, G., \& Bastien, P. 2006, A\&A, 459, 797

Pinte, C., Ménard, F., Berger, J., Benisty, M., \& Malbet, F. 2008a, ApJ, 673, L63 Pinte, C., Padgett, D. L., Ménard, F., et al. 2008b, A\&A, 489, 633

Pinte, C., Harries, T. J., Min, M., et al. 2009, A\&A, 498, 967

Pollack, J. B., Hollenbach, D., Beckwith, S., et al. 1994, ApJ, 421, 615

Sitko, M. L., Carpenter, W. J., Kimes, R. L., et al. 2008, ApJ, 678, 1070

Tatulli, E., Millour, F., Chelli, A., et al. 2007, A\&A, 464, 29

van den Ancker, M., The, P., Tjin A Djie, H., et al. 1997, A\&A, 324, L33 van der Plas, G., van den Ancker, M. E., Acke, B., et al. 2009, A\&A, 500, 1137 Varnière, P., Blackman, E. G., Frank, A., \& Quillen, A. C. 2006, ApJ, 640, 1110 Vinković, D., Ivezić, Ž., Jurkić, T., \& Elitzur, M. 2006, ApJ, 636, 348 